\title{
NINETEENTH-CENTURY INDIAN PLACE \\ NAMES WITH CONTEMPORARY VARIANTS \\ AND TWENTY-FIRST-CENTURY \\ EQUIVALENTS
}

\begin{tabular}{|c|c|c|}
\hline $\begin{array}{l}\text { Nineteenth-century } \\
\text { name }\end{array}$ & $\begin{array}{l}\text { Nineteenth-century } \\
\text { variants }\end{array}$ & $\begin{array}{l}\text { Twenty-first-century } \\
\text { name }\end{array}$ \\
\hline Anamagalam & & Annamagalam \\
\hline \multicolumn{3}{|l|}{ Andimadam } \\
\hline Arkonam & & Arakkonam \\
\hline Bancoorah & & Bankura \\
\hline Bangalore & Bangalur & Bengaluru \\
\hline Baroda & & Vadodara \\
\hline Benares & & Varanasi \\
\hline Betegeri & $\begin{array}{l}\text { Betgeri/Bettighery/ } \\
\text { Bettigeri }\end{array}$ & Gadag-Betageri \\
\hline \multicolumn{3}{|l|}{ Bodinayakanur } \\
\hline Bombay & & Mumbai \\
\hline Burdwan & & Bardhaman \\
\hline Bussahir & & Bushahr \\
\hline Calcutta & & Kolkata \\
\hline Calicut & & Kozhikode \\
\hline Cannanore & Cannanur/Kannanur & Kannur \\
\hline Cape Comorin & & Kanyakumari \\
\hline Ceylon & & Sri Lanka \\
\hline Chinsurah & & Hooghly \\
\hline Chota Nagpur & Chotanagpur & Chota Nagpur \\
\hline Coorg & Kurg/Kurgland & Kodagu \\
\hline Dhawar & Dharward & Dharward \\
\hline Dindigal & & Dindigul \\
\hline Gorockpur & & Gorakhpur \\
\hline Hoobly & & Hubali \\
\hline
\end{tabular}


TWENTY-FIRST-CENTURY EQUIVALENTS

\begin{tabular}{|c|c|c|}
\hline $\begin{array}{l}\text { Nineteenth-century } \\
\text { name }\end{array}$ & $\begin{array}{l}\text { Nineteenth-century } \\
\text { variants }\end{array}$ & $\begin{array}{l}\text { Twenty-first-century } \\
\text { name }\end{array}$ \\
\hline Jeypur & Jaipur & Jeypore \\
\hline Kaimbatur & & Coimbatore \\
\hline Kairkal & & Karaikal \\
\hline Kalashti & & Srikalahasti \\
\hline Kanara & Canara/Karnataka & $\begin{array}{l}\text { Kanara/Canara/ } \\
\text { Karnataka }\end{array}$ \\
\hline Keti & Kety/Kaity & Ketti \\
\hline Kotageri & & Kotagiri \\
\hline Kudelur & & Cuddalore \\
\hline \multicolumn{3}{|l|}{ Kumbakonam } \\
\hline Lahore & & Lahore \\
\hline Madras & & Chennai \\
\hline Madura & & Madurai \\
\hline Mayavang & & Mayiladuthurai \\
\hline \multicolumn{3}{|l|}{ Mannargudi } \\
\hline Mangalore & Mangalur & Mangalore \\
\hline Mayavaram & & Mayiladuthurai \\
\hline Mercara & & Madikeri \\
\hline \multicolumn{3}{|l|}{ Motupatti } \\
\hline Nayudupetta & & Naidupeta \\
\hline Negapatam & & Nagapattinam \\
\hline Nilgiri & Nilgiris/Nilagiri & Nilgiri \\
\hline \multicolumn{3}{|l|}{ Palghat } \\
\hline Panrutti & & Panruti \\
\hline Pegu & & Bago \\
\hline Peshawar & & Peshawar \\
\hline Pollachi & & Palakkad \\
\hline Pondicehri & & Puducherry \\
\hline Pudukotei & & Pudukkottai \\
\hline Puna & Poona & Pune \\
\hline Ramandroog & Ramandrog & Ramandroog \\
\hline
\end{tabular}


TWENTY-FIRST-CENTURY EQUIVALENTS

Nineteenth-century

name

Sadras

Sengelpa

Sholapur

Sidambaram

Sind

Sivaganga

Tandshur

Taliparambu

Tanjore

Talatscheri

Tinnevelly

Tirhoot

Tirumangalam

Tirupatur

Tiruppur

Triwallur

Tranquebar

Trichinopoli

Tripumtura

Tuticors

Udapi

Udumalpet

Umballa

Utakamand

Vilupuram

Virudalpati

Virrutasalem

Yercard
Nineteenth-century variants

Twenty-first-century name

Chengalpattu

Shalapoor

Solapur

Chidambaram

Sindh

Taliparamba/

Thaliparamba

Thanjavur

Thalassery

Tirunelveli

Tirhut

Tirupattur

Tiruwalur

Trichinopoly

Udipi

$\begin{array}{ll} & \begin{array}{l}\text { Ambala } \\ \text { Udhagamandalam/ } \\ \text { Ootacamund/ } \\ \text { Udhagai/Ooty } \\ \text { Viluppuram }\end{array} \\ \text { Villupuram } & \text { Virudhachalam } \\ & \text { Yercaud }\end{array}$

[ $\mathrm{xx}$ ]
Tiruvallur

Tharangambadi

Tiruchirappalli

Thrippunithura

Tuticorin/

Thoothukudi

Udupi

Ambala

Udhagamandalam/

Ootacamund/

Uiluppuram

Yercaud 